\title{
Induced subject-relative movement: Persistence of apparent movement of a stationary point after removal of inducing stimulus
}

\author{
R. H. DAY, R. G. DICKINSON, and K. I. FORSTER \\ Monash University, Clayton, Victoria, 3168, Australia
}

\begin{abstract}
It was observed by chance that perceived movement of a stationary spot of light in a dark featureless field persists after its induced movement by a moving frame. When the frame was suddenly occluded, apparent movement of the spot persisted in the same direction as prior induced movement. The effect which is compelling and readily reported and referred to as induced subject-relative movement (ISRM) was confirmed and further investigated in four experiments. In the first, the informal observations of ISRM were confirmed using manual tracking to index perceived movement, and in the second, it was shown to occur only very slightly and briefly when the frame merely stopped. In the third experiment, ISRM was shown to occur following two different paths of induced movement, and in the fourth, not to occur following real movement of the spot, which was almost indistinguishable from its induced movement. It is suggested that the effect arises from the absence of a signal for cessation of perceived movement when the frame disappears.
\end{abstract}

The purpose of this paper is to describe a new visual illusion of movement, apparent movement of a stationary point of light in an otherwise dark field in the same direction as its immediately preceding induced movement. The effect was first observed by chance during experiments on induced movement when a rectangular frame moving laterally and enclosing a small light spot was suddenly occluded. The spot appeared to continue moving in the same direction as its preceding induced movement for an appreciable period but with diminishing apparent velocity. The effect which is perceptually continuous with induced movement has proved to be extraordinarily compelling for almost all observers, is readily and instantly reported, and, as far as can be ascertained, has not been described before. Four formal experiments and some preliminary observations following induced movement in a circular path are described.

An effect which is superficially similar, but opposite in direction, to that reported here was described by Brosgole (1967). The egocentric location of a stationary spot of light was apparently shifted from its straightahead position by moving a surrounding frame laterally. The frame was then occluded. The spot appeared to drift back to its original position. This drift was opposite to its earlier direction and in the same direction as the real movement of the frame. However, Brosgole used a "cancellation" method which involved the subjects in controlling the position of the spot to maintain it throughout in the apparently straight-ahead position. Thus the spot actually moved as the subject controlled it, and this real movement was superimposed on induced

Requests for reprints should be addressed to Professor R. H. Day, Department of Psychology, Monash University, Clayton, Victoria, 3168, Australia. This work was supported by Grant A65/15940 from the Australian Research Grants Committee. movement and apparent drift. In the experiments described here, the spot was always stationary and straight ahead while the subject tracked its perceived motion manually. The two stimulus situations were therefore different.

The phenomenon to be described can be considered from various standpoints. It can be regarded as a special case of autokinetic movement, the speed and direction of which are determined by prior induced movement. Alternatively, it can be treated as a special instance of induced movement which persists after removal of the inducing field. Again, the illusion might be unrelated to either effect. To avoid prejudicing the issue at this stage, the effect will be called simply induced subject-relative movement, adapting the term used by Shaffer and Wallach (1966), and referred to as ISRM. Induced movement will be referred to as IM and real movement as $\mathrm{RM}$.

The four experiments were conducted after 10 observers, who did not participate again, informally confirmed the initial chance observation of the effect verbally. The first experiment was simply concerned with further confirmation of the effect using a manual tracking technique, the second with controlling for its possible occurrence when the frame remained visible after it stopped, the third with the effects of different IM paths within the frame, and the fourth with its possible occurrence following RM of the point in the stationary frame. A control for the possible occurrence of ISRM before frame occlusion was also included in the third and fourth experiments.

\section{EXPERIMENT I}

The purpose of the first experiment was to confirm 
the earlier informal observations and verbal reports of ISRM under more controlled conditions and to record it by means of manual tracking.

\section{Method}

Subjects. There were 12 subjects, 8 women and 4 men, all of whom were student volunteers paid at the rate of $\$ 1 / \mathrm{h}$. None had had prior experience of the effect under investigation.

Apparatus. The viewing distance throughout was $257 \mathrm{~cm}$ and was controlled by means of a chinrest. The stimulus array consisted of a light spot, which was always stationary and straight ahead of the subject at eye level, and a coplanar, lighted, rectangular frame which surrounded it. The circular spot was $.3 \mathrm{~cm}$ in diameter (visual angle: $.07^{\circ}$ ). The border of the frame was $.5 \mathrm{~cm}\left(.11^{\circ}\right)$ wide. In outside dimensions, the frame was $30 \mathrm{~cm}$ $\left(6.65^{\circ}\right)$ horizontally and $20 \mathrm{~cm}\left(4.43^{\circ}\right)$ vertically. The luminance of spot and frame was $1 \mathrm{~cd} / \mathrm{m}^{2}$. The frame could be moved left or right at $2.26 \mathrm{~cm}\left(.5^{\circ}\right) / \mathrm{sec}$ by a motor operating through a rack and pinion linkage. The motor continued to operate throughout the trial irrespective of whether the moving frame was visible or occluded. ${ }^{1}$ Black draw-curtains in front of the stimulus array prevented any view of the apparatus or stimulus pattern when the subject entered the room and between trials when the room lights were switched on. During a trial, only the sharply defined stimulus elements were visible in the otherwise dark laboratory.

A manual tracking device that had proved satisfactory earlier in recording a visual movement aftereffect (Day \& Strelow, 1971; Strelow \& Day, 1971, 1975) was used throughout. A carriage that was mounted above a moving $(.70 \mathrm{~cm} / \mathrm{sec})$ chart was moved by the subject from side to side at right angles to chart movement to match direction and speed of apparent movement of the spot. A permanent record of this manual response was traced on the chart. The instant at which the frame was occluded by switching off its separate light source was also recorded on the chart. The tracking device was placed on a table at which the subject sat throughout the experiment. The device was located to the subjects's right and was operated with the right hand.

Manual tracking and perceived velocity. The use of manual tracking to reproduce visual movement raises the question of the relationship between perceived visual velocity and speed of lateral hand movement. Can it be assumed that hand movement accurately reproduces visually perceived velocity? There is a difficulty in testing this assumption directly for induced movement. The physical velocity of the moving inducing element is perceptually "shared" between it and the stationary target. Without an independent measure of perceived target velocity, it is not possible to establish the accuracy with which it is reproduced by manual tracking. However, there is good evidence to show that real movement (RM) of a display element in a well-structured visual field can be accurately reproduced. Strelow and Day (1975) required 24 subjects to track manually vertical dark and light bars moving from side to side in a circular aperture subtending $3^{\circ}$ at the center of a circular $29^{\circ}$ patterned field. The velocity of the bars was $5.4 \mathrm{~mm} / \mathrm{sec}$, the viewing distance $157 \mathrm{~cm}$, and the tracking apparatus that described above. The average speeds of tracking over two trials for the 24 subjects were 5.40 and $5.65 \mathrm{~mm} / \mathrm{sec}$, virtually a $1: 1$ ratio between target and hand speeds. Since there is known to be a close relationship between real and perceived velocity in this range (Mashhour, 1964), it is reasonable to assume that the speed of manual tracking accurately reflects visually perceived speed and, therefore, to regard manual tracking speeds as reflecting perceived velocities.

Procedure. An experimental session consisted of instructions, familiarization, tracking practice and four experimental trials. The instuctions simply directed the subject to track the spot when it was seen to be moving by moving the carriage in the appropriate direction and at the same speed, and to hold the carriage still when the spot was seen as stationary. No reference was made to apparent or illusory movement. Each subject then underwent a familiarization and tracking practice trial, during which only IM was demonstrated and tracked. In the familiarization and practice trials, the subject, with head positioned, observed the spot during a $25 \mathrm{~cm}\left(5.54^{\circ}\right)$ excursion of the frame and then tracked it for another. In the experimental trials which followed, the subject again tracked apparent movement of the stationary spot, but when the frame had traveled half its disance, i.e., $12.5 \mathrm{~cm}\left(2.77^{\circ}\right)$, it was occluded by switching off its separate light source. The frame took $11.1 \mathrm{sec}$ to travel $25 \mathrm{~cm}$ and was thus visible for $5.6 \mathrm{sec}$. At the beginning of each trial, the spot was near the left or right vertical edge of the frame, and when the frame was occluded, at its center. During two trials, the frame moved from right to left (L), and during the other two, from left to right (R). Half the subjects underwent the four trials in the order RLLR, and the other half in the order LRRL.

The records were scored in terms of tracked velocity by measuring the distance moved by the hand during each second for the 5 -sec periods immediately before ( $\mathrm{MM}$ phase) and after (ISRM phase) occlusion of the moving frame.

\section{Results}

To compare velocities between the two phases and two directions of movement, the mean velocities for the $5 \mathrm{sec}$ over each phase were found for each subject. These means were derived from the two trials in the one direction. An analysis of variance of these means showed that the main effect for Phase was significant, $F(1,11)=120.51, p<.005$, but that for Direction of Movement was not, $F(1,11)=2.88, p>.05$. The interaction between Phase and Direction was significant, $F(1,11)=5.50, p<.05$.

Mean velocities of apparent movement of the spot based on the four trials for the 12 subjects are shown second by second for each phase in Figure 1. Standard errors are also shown. The values are plotted at the midpoint of each second. It can be observed that when the moving frame was visible the velocity of the IM was about $14-18 \mathrm{~mm} / \mathrm{sec}$. After occlusion of the frame, apparent movement (ISRM) continued but declined sharply in velocity over the $5 \mathrm{sec}$. Mean velocity at the end of the second 5 -sec period was about $3 \mathrm{~mm} / \mathrm{sec}$. Examination of individual records showed that all 12 subjects perceived ISRM. Of the 48 records, 47 showed apparent movement of the spot in the same direction as IM. The direction of the remaining record was negative, i.e., in the opposite direction. It is of interest to note also that about half the subjects commented on ISRM about $10 \mathrm{sec}$ or more after occlusion of the frame.

In general, the results from the first experiment show that ISRM consistently occurs following IM of the spot, its direction is the same as that of IM, and its velocity declines sharply over a period of about $5 \mathrm{sec}$.

\section{EXPERIMENT II}

Since the initial observations were made following sudden occlusion of the frame, it was assumed that IM with spot and frame resulted in ISRM when the frame was removed and the spot alone remained visible in the dark field. No attention was given to the possibility 
that IM of the spot might persist when the frame was stopped but remained visible. However, informal observations indicated that no such persistence of spot movement occurred. Nevertheless, it was possible that some apparent movement of the spot might have resulted from suggestion associated with lateral hand movements. The second experiment was essentially the same as the first, except that after a period of IM of the spot the movement of the frame was merely stopped.

\section{Method}

Subjects. There were 12 subjects, 7 women and 5 men from the same population as those in the first experiment.

Procedure. The only difference between the procedure of this and the first experiment was that the frame was suddenly stopped rather than occluded as during the four experimental trials. The sound of the motor continued throughout. Instructions, familiarization, tracking practice, and scoring were the same as in Experiment I.

\section{Results}

Mean tracked velocities of the stationary spot during the $5 \mathrm{sec}$ periods before and after cessation of frame movement are plotted in Figure 2. It can be 'seen that apparent movement of the frame persisted for about 1 sec after the frame stopped, but thereafter ceased altogether. Mean velocity during the first second was about $4 \mathrm{~mm} / \mathrm{sec}$, compared with about $9 \mathrm{~mm} / \mathrm{sec}$ for the same period in the first experiment. This brief persistence was unexpected. Two possible reasons for

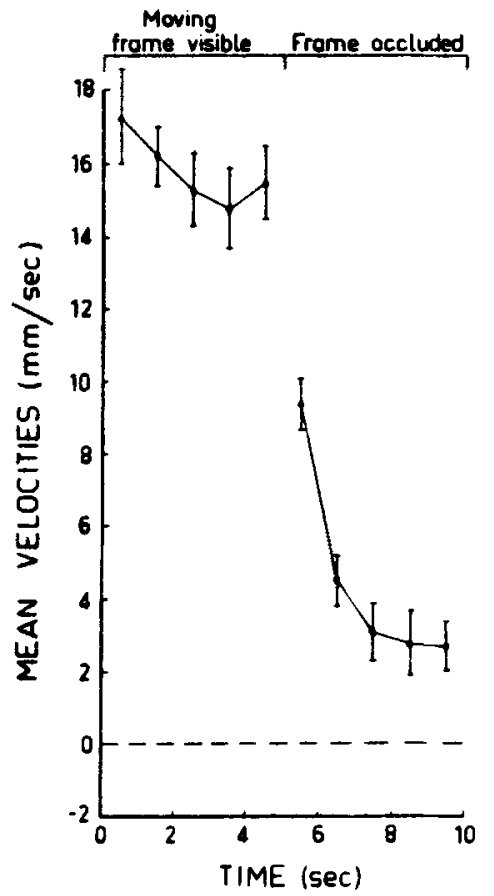

Figure 1. Mean tracked velocities of the stationary light spot when it was enclosed in the moving frame (induced movement) and by itself in a dark field after occlusion of the frame (induced subject-relative movement) in Experiment 1. The mean velocities are plotted at the midpoint of each second of the 10-sec trial. Standard errors of the means are shown as vertical bars.

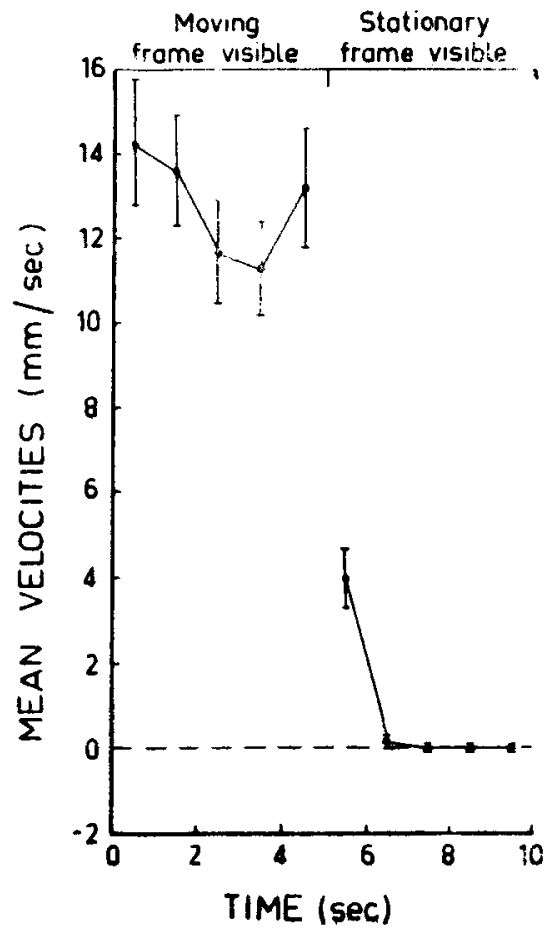

Figure 2. Mean tracked velocities of the stationary light spot when it was enclosed in the moving frame and the stationary frame in Experiment II. Standard errors of the means are also shown.

its occurrence can be suggested. Given the nature of the tracking task and uncertainty in judging when a slowly moving element stops, it is conceivable that the $1 \mathrm{sec}$ of apparent movement simply represents a reaction time to cessation of movement. On the other hand, there might be slight ISRM when the frame stops but remains visible. As will be seen, the data from a later experiment (Experiment IV) favor the second of these possibilities. Briefly, in the later experiment, there was no indication of a reaction time to cessation of real movement of the spot, which is hardly distinguishable from its $\mathbf{M}$, and the basis of ISRM seems to be the absence of a signal for cessation of apparent movement. This latter condition obtained to some extent in the second experiment. This point is taken up again in the concluding discussion.

\section{EXPERIMENT III}

The data from Experiment I showed that ISRM of the spot by itself followed its IM with the moving frame. Although casual observation did not suggest that movement of the spot alone occurred prior to $\mathrm{IM}$ or persisted from an earlier trial, there was no formal control for these possibilities. Moreover, it was conceivable that manual tracking from side to side might also have suggested some horizontal apparent movement of the spot in the featureless field. In the third experiment, therefore, the spot by itself was tracked for $5 \mathrm{sec}$ both before and after IM with the moving frame. 


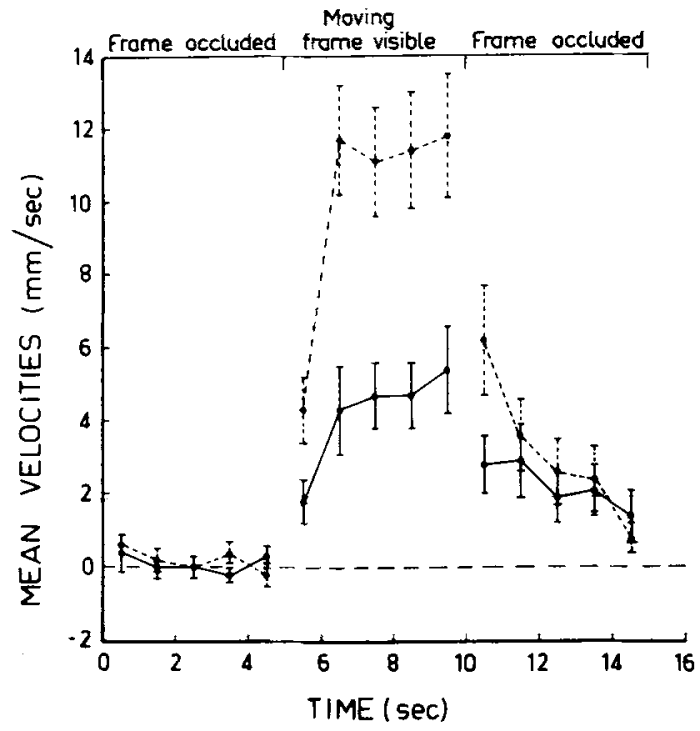

Figure 3. Mean tracked velocities of the stationary light spot before (control) and after (induced subject-relative movement) its induced movement in the moving frame for two paths of induced movement, center to edge (dotted line) and edge to center (continuous line) in Experiment III. Standard errors of the means are also shown.

The path of IM was constant in Experiment I; the dot was initially located near a vertical edge of the frame, and when the latter was occluded it was at its center. Occlusion of the frame when the stationary spot was at its center, i.e., half way across the visible field, might have suggested continuation of apparent movement. In other words, continuation of spot movement could have arisen from occluding the inducing field when the spot appeared to have traveled only half the distance across it. If this were so, then ISRM would not be expected to occur, or to occur only weakly, when the path of IM of the spot was from the center to the boundary of the frame. Another reason for varying the path of the spot in the IM phase was that in Brosgole's (1967) experiment, in which an effect opposite in direction to that obtained in Experiment I occurred, the frame was occluded when the spot was near its edge. Although Brosgole's methods were different, as pointed out above, his results nevertheless suggested that the path of IM might affect the direction of ISRM.

\footnotetext{
Method

Subjects. There were two new groups of 10 subjects, all volunteers from the same population and paid for their services as in Experiments I and II. There were 5 women and 5 men in one group and 4 women and 6 men in the other.

Apparatus. The stimulus array was slightly modified by decreasing the horizontal extent of the frame from 30 to $25 \mathrm{~cm}$ $\left(5.54^{\circ}\right)$ in order to accommodate an additional 5 -sec period in the total period of horizontal travel of the frame. ${ }^{2}$ The apparatus was otherwise the same as in the earlier experiment.

Procedure. Following instructions, there were four trials, each consisting of three 5 -sec phases, the first serving as a control for the third. During the first and third phases, the spot alone was visible, and in the second, the spot and moving frame together.
}

In order to standardize the instructions and to keep them uniform throughout, they were recorded and played to the subject. The content was the same as in Experiment I. Familiarization and practice trials were not included. They were omitted because of a remote possibility that by showing subjects IM it might be unduly emphasized to the possible exclusion of attention to ISRM.

For one group, the path of IM during the second 5 -sec period was from the inner vertical edge of the frame to the center and for the other from center to edge. Thus, for the first group, the frame was occluded when the spot was at the center of the frame and for the other when it was nearly at the inside boundary. Apart from these changes, the procedure was the samethroughout as in Experiment $I$, including the number of tials per subject (4), directions of frame movement ( $R$ and ${ }^{\prime} L$ ), and scoring. Each score was the mean tracked velocity, second by second, derived from the two trials in one direction.

\section{Results}

Mean tracked velocities over the three 5-sec phases for the two groups, each based on four trials per subject (direction was again not significant), are shown in Figure 3. It can be seen that, while apparent movement during the first $5 \mathrm{sec}$ was virtually negligible, it clearly occurred during the last $5 \mathrm{sec}$ of the trial. That is, ISRM occurred only following IM. It can be noted also that the direction of ISRM was again in the same direction as IM for both groups. Inspection of the graph also indicates that there was a difference in velocity of IM according to its path in the frame and that the velocity of ISRM was initially de termined by that of IM.

As in the first experiment, the mean velocities for each subject over the two 5-sec periods were found and compared in an IM path (2) by direction (2) by phase (3) analysis of variance. This showed that the main effects for IM path and phase were significant, $F_{\text {path }}(1,18)=6.50, \quad p<.025 ; \quad F_{\text {phase }}(2,36)=63.57$, $p<.055$, but that for direction was not $(F<1)$. The only interaction to reach significance was that between IM path and phase, $F(2,36)=13.21, p<.005$. Further analysis using planned comparisons indicated that there was no difference between tracked velocities for the two groups in the first (control) phase and in the third (ISRM) phase ( $F<1$ in each case), but that there was a significant difference in the second (IM) phase, $F(1,36)=44.20, p<.055$. That is, the velocity of IM varied with its path.

Although the average velocity over the whole 5 -sec period for the two groups in the last phase was not significant, suggesting that ISRM velocity is not determined by that of IM, inspection of Figure 3 suggests that this conclusion is not entirely warranted. It can be seen that initially there was a difference between tracked velocities, each corresponding with its preceding IM velocity, but that they converged towards the end of the period. Therefore, mean velocities for each second of the last phase, each based on four trials, were compared in a stage (5) by group (2) analysis. This showed that the main effect for stage was significant, $F(4,72)=17.04$, $p<.005$, but that for groups was not $(F<1)$. However, the interaction between stage and group achieved significance, $F(4,72)=10.33, p<.005$, suggesting that the 
difference between the two groups was significant in the early stages of the $5 \mathrm{sec}$ period. It seems reasonable to conclude, therefore, that perceived velocity of IM determines that of ISRM at the beginning of the latter phase but that this difference is lost towards the end of it.

Since ISRM is relatively long lasting, the possibility existed that the residual effect from one trial might persist and sum with the effect in the next. Inspection of the data for individual subjects during the first phase of the four succeeding trials ruled out this possibility. These data show that in almost every case there was no apparent movement of the spot during the first phase and that there was no tendency towards an increase in apparent movement over successive trials.

The results from the third experiment show that ISRM occurs only after IM and that the tracked velocity of IM varies with the path of apparent movement in the moving frame, with the center to boundary path resulting in greater velocity than that from boundary to center. Furthermore, the velocity of ISRM is initially determined by that of IM. Finally, there is no tendency for ISRM to persist sufficiently to sum with that in subsequent trials.

\section{EXPERIMENT IV}

Informal observations have shown that IM of a small stationary spot in a moving frame in an otherwise dark environment is hardly distinguishable from $R M$ of the same spot in a stationary frame. Therefore, if ISRM is simply persistence of perceived movement of a target following its apparent relative movement, it would be expected also to occur following its real movement. The fourth experiment was designed to establish whether ISRM follows RM of the spot in a stationary frame. However, an IM condition was included so that ISRM, if it occured after RM, could be compared with that following $\mathrm{IM}$. The inclusion of an IM condition was considered necessary in any case, since new apparatus was constructed to enable separate movement of spot and frame while the other element remained stationary.

\section{Method}

Subjects. There were 16 subjects, all volunteers and drawn from the same population as the earlier experiments. There were 10 men and 6 women. The subjects were paid for their participation at the same rate as in the earlier experiments.

Apparatus. The apparatus used in the three earlier experiments permitted movement of the frame only while the spot remained stationary. Since the RM situation in this experiment required movement of the spot while the frame was stationary, it was necessary to construct new apparatus. However, although this apparatus was differently constructed and hydraulically operated, its display was essentially the same as in the three earlier experiments. The dimensions of the frame and dot were the same as for Experiment III but their luminance and speed were slightly less. The luminance of both elements was $.5 \mathrm{~cd} / \mathrm{m}^{2}$, and each could be moved either left or right at $.45^{\circ} / \mathrm{sec}$ compared with $1 \mathrm{~cd} / \mathrm{m}^{2}$ and $.5^{\circ} / \mathrm{sec}$ in the other three experiments. The viewing distance throughout was $257 \mathrm{~cm}$. All other features of the experimental situation were the same as in Experiment III.

Procedure. As in the third experiment, there were three 5 -sec phases in each trial, the first serving as a control for the third. During the first and third phases, the spot alone was visible and in the second, the spot and frame together. The task throughout was to track apparent movement of the spot during each $15 \mathrm{sec}$ trial.

There were four conditions, induced (IM) and real (RM) movement of the spot during the second phase and movement of the spot from the center to the edge of the frame (CE) and from edge to center (EC) during the same phase. During IM, the spot was stationary while the frame moved, and during RM, the spot moved while the frame was stationary. The path of the spot in the frame was again varied, since this had proved to be a significant variable in Experiment III and interest attached to whether apparent velocity woukd vary similarly with $R M$.

There were four trials under each condition. Each subject participated under all four conditions, completing the four trials in one condition before proceeding to those in the next. The order in which the four conditions were presented was varied by selecting 8 of the 24 possible orders. Two subjects were presented with the same order, but for one the apparent direction of spot movement in the frame was left to right and for the other right to left.

The instructions that were again played were those used in the third experiment. Since apparent direction of movement had not proven to be significant in earlier experiments, the second-by-second scores for each subject were the mean tracked velocities over four trials.

\section{Results}

Mean tracked velocities over the three 5-sec phases for the four conditions, each based on four trials per subject, are shown in Figure 4. Because inclusion of standard errors in the graph would make it difficult to read, they have been omitted. However, their order of magnitude were about the same as that in Figure 3. It can be seen from Figure 4 that apparent velocity for CE movement of the spot was greater for both IM and RM than for EC movement, the result for IM confirming that obtained in Experiment III. The most striking feature of Figure 4, however, is a complete absence of ISRM following RM of the spot. Not only was ISRM absent, but apparent movement of the spot ceased immediately the frame was occluded. There is no suggestion of any "lag" in the cessation of movement as occurred in Experiment III.

An overall analysis of the data involving the three phases was considered unnecessary. Instead, three separate analyses, one for each phase, were carried out The mean velocities shown in Figure 4 were averaged over the 5-sec phases, and these means statistically examined in a movement path (2) by movement type (2) analysis of variance for each phase. There were no significant differences between the means for the first $5 \mathrm{sec}$ (all $F$-ratios were less than 1 ), an outcome which is not surprising, since the conditions were uniform throughout for this control phase. In the second phase, the difference between movement paths $\mathrm{CE}$ and $\mathrm{EC}$ was significant, $F(1,15)=6.79, p<.025$, together with that between $\mathrm{IM}$ and $\mathrm{RM}, F(1,15)=6.23, p<.025$. The interaction between these two variables did not achieve significance, $F(1,15),=2.69, p>1$. In the third 
phase, the difference in ISRM following IM and RM was significant, $F(1,15)=9.56, p<.01$, but that following $\mathrm{CE}$ and $\mathrm{EC}$ movement was not $(F<1)$. The interaction between the two variables also failed to reach significance $(F<1)$.

These results are convincing in showing that al though IM and RM of a spot of light in an enclosing frame are perceptually difficult to distinguish, ISRM does not follow the latter. The effect seems to follow only apparent movement of the stationary spot, which is induced by movement of its surrounding frame. A possible reason for this is suggested below.

\section{DISCUSSION}

Taken together, the results from the four experiments show that, following induced movement (IM) of a stationary spot of light in a moving frame, the spot alone in a dark featureless field appears to continue moving in the same direction but with diminishing apparent speed. Such apparent movement of the stationary element, here called induced subject-relative movement (ISRM); occurs also, but only very slightly and briefly, when the inducing frame merely stops but remains visible and not at all following real movement (RM) of the spot, which is hardly distinguishable perceptually from IM. The effect was readily observed and responded to by all subjects on almost all trials. It is a vivid and compelling phenomenon. In phenomenal terms, the apparently moving spot simply continues to move when the frame suddenly disappears. There is no marked qualitative difference between IM and ISRM; the second is perceptually continuous with the first.

Before considering theoretical and other implica-

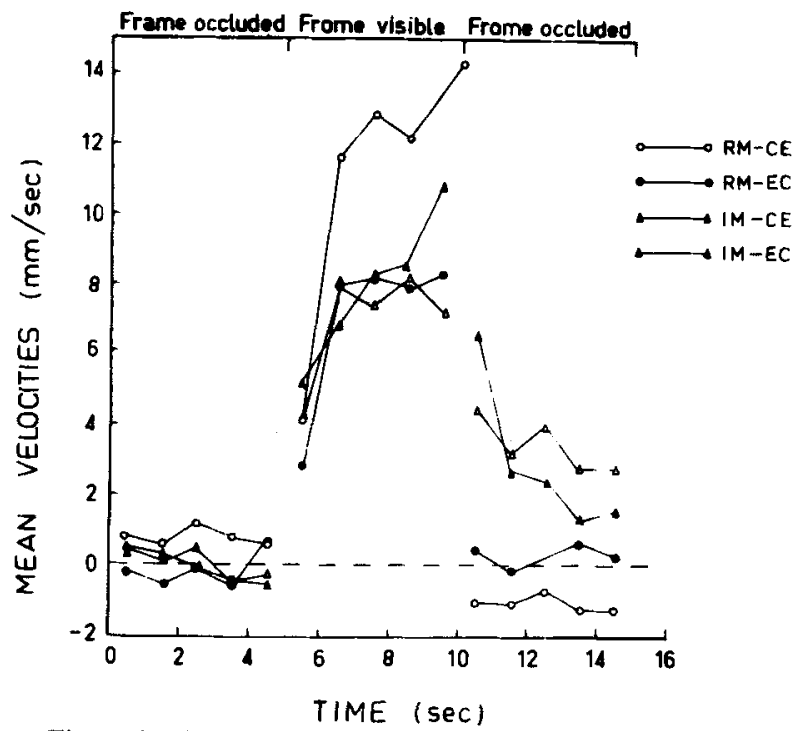

Figure 4. Mean tracked velocities of the stationary spot before (control) and after (ISRM) its induced movement (IM) and real movement $(R M)$ from center to edge $(C E)$ or edge to center (EC) of the frame in Experiment IV. During IM of the spot, the frame moved, but during $R M$, it was stationary. tions of ISRM, some aspects of IM and RM which have emerged incidentally from the experiments deserve brief comment. These are the effect of IM and RM path (center to edge, edge to center) on their apparent velocity, the different magnitudes of IM velocity and its different trends in the four experiments.

It is clear from Experiments III and IV (Figures 3 and 4) that IM is significantly affected by its path of movement in the frame and from Experiment IV that RM is similarly affected. Apparent velocity from the center to the edge of the frame is greater than that from edge to center. In suggesting a possible explanation of this difference, two points from a recent experiment need to be made clear. First, depending on which element is attended to, the spot and the frame appear to move when the latter actually does so. Second, RM of the spot produced IM of the frame, although at a markedly lower apparent velocity. Given these facts, it is reasonable to assume that RM of a stimulus feature is perceptually "shared" between that feature and another which is adjacent and stationary. If such sharing of the movement of one element between two occurs, then it is reasonable to suppose that it does so most readily when the two elements are initially close together, as in the EC condition. When initially they are widely separated, as in the $\mathrm{CE}$ condition, there is less sharing so that the apparently moving element appears to be traveling faster. That is, the total velocity is more fully shared when the two are initially adjacent so that the individual velocity of that attended to is less than when it is initially widely separated from the other. While conjectural, such a notion of sharing and monopolizing of RM by adjacent and removed elements, respectively, offers a testable basis for the difference found in Experiment III for movement paths and confirmed in Experiment IV.

A second feature of IM is the variability of its apparent velocity and of the trend in its velocity during 5 sec. In Experiments I and II, mean apparent velocity varied between about 11 and $17 \mathrm{~mm} / \mathrm{sec}$, and in Experiments III and IV, between about 10 and $12 \mathrm{~mm} / \mathrm{sec}$. In the first two experiments, there was also a tendency for velocity to decline during the 5-sec period, and in the last two, for it to increase slightly. Neither of these differences can be readily explained. However, it can be noted that there were two differences between the first and last two experiments. In Experiments I and II, the instructions were given by the experimenter to the subject, whereas in Experiments III and IV, they were recorded and played. Second, in the first two experiments there was no initial control period during which the spot alone was tracked as there was in the second two. While the differences in magnitude and trend of IM cannot on the evidence be attributed solely to these differences in procedure, it is nevertheless reasonable to suppose that IM is sensitive to such instructional and procedureal variables. Further experiments are necessary to establish the effect of such variables. 
The occurrence of apparent movement of a stimulus element by itself in a featureless field following its induced movement by a moving field can be usefully considered from the standpoints of the visual movement aftereffect, autokinetic movement, and the absence of the effect following RM. As will be seen, the difference between the outcomes for IM and RM sug. gests a possible basis for the ISRM effect.

Is ISRM a special case of a visual movement aftereffect for which the prior condition is induced rather than real movement? The answer must be in the negative. In the first place, the motion aftereffect is typically in the opposite direction to preceding movement. The effect described here was in the same direction as preceding induced movement of the spot. Second, the motion aftereffect either does not occur at all or occurs only very weakly in the absence of a patterned surround. Although the surround might be present during induction, its absence in the aftereffect reduces the effect considerably (Day \& Strelow, 1971; Strelow \& Day, 1975). It seems unlikely, therefore, that ISRM represents a special instance of an aftereffect.

Autokinetic movement is a form of illusory subjectrelative movement which occurs most commonly after a latency period with a single point of light in a dark featureless field. It is variable in direction and extent, often reported as "wandering," and its direction is affected by a wide range of stimulus and subject variables (Graybiel \& Clark, 1945; Royce, Carr, Aftanas, Lehman, \& Blumenthal, 1966). There is hardly any data relating to the effect of prior movement of the target on autokinetic movement. However, Hoffman, Swanden, Baran, and Rohrer (1953) found that the judged extent of autokinetic movement varied as a function of repeated prior exposure to an actually moving light.

Can ISRM be regarded as a particular instance of autokinetic movement the latency of which is reduced or eliminated and the initial direction and speed of which is determined by prior movement? The answer to this question is probably negative also. If the effect were a special case of autokinesis, with prior perceived movement in the frame eliminating its latency and determining its direction and speed, then it would be expected to occur following $\mathrm{IM}$ and $\mathrm{RM}$, which are perceptually very similar. The fact that ISRM does not occur following RM would seem to rule out this possibility.

The consistent occurrence of ISRM following IM and its complete absence following RM, which is perceptually almost indistinguishable, is among the most striking outcomes of the experiments. Is there a difference between IM and RM which can account for this? The most likely difference seems to be that between the two forms of apparent movement at the moment the frame is occluded. At this point, during IM, the apparently moving but physically stationary spot remains stationary but continues perceptually to move. When occlusion occurs during RM, the apparently and physically moving spot stops in both respects. Thus there is no change in the physical status of the spot at the point of frame occlusion during IM, while there is a change from movement to stationariness in the case of RM. This difference, and its perceptual outcome, raises the interesting issue concerning the perceptual basis of the cessation of perceived movement. When movement is induced and the inducing element is suddenly removed, there is no change in the physical state of the apparently moving element which continues to appear to move. It is conceivable that ISRM occurs because there is no signal for its cessation. On the other hand, there is such a signal, a change from movement to stationariness, in the case of RM. In brief, a possible basis for ISRM is the absence of any signal which indicates that the status of the apparently moving but physically stationary element has changed.

The data from Experiment II can be construed as supporting the argument that ISRM results from the lack of a stimulus for cessation of movement of the spot. In this experiment, there was a slight and brief (about $1 \mathrm{sec}$ ) ISRM when the frame stopped but remained visible. It is conceivable that this brief episode was also occasioned by the absence of any change in the status of the spot itself when the frame stopped. Longer persistence of the effect would have been prevented by absence of any motion relative to the frame itself. Incidentally, the brief and slight effect in Experiment II suggests that the signaling of the cessation of perceived movement may derive from the target object itself as well from its relationship to adjacent stationary objects.

Finally, does ISRM occur also following nonlinear induced movement? A series of informal observations has shown that it does. A square frame surrounding a stationary spot moved in a circular path. This resulted in a compelling impression of the spot moving around a circular path in the stationary square. When the square was occluded, subjects reported a persistence of movement along the same path. However, reports of movement in the opposite direction to IM were common. Further investigation of ISRM under these conditions is necessary.

\section{REFERENCES}

Brosgole, L. Induced autokinesis. Perception \& Psychophysics, 1967, 2, 69-73.

DAY, R. H., \& Strelow, E. R. Visual aftereffect of movement: Partial or complete reduction in the absence of a patterned surround. Nature, 1971, 230, 55-56.

Graybiel, A., \& Clark, B. The autokinetic illusion and its significance in night flying. Journal of Aviation Medicine, 1945, 16, 111-151.

Hoffman, E. L., Swanden, D. V. K., Baran, S. H., \& ROHRER, J. H. Generalization and exposure time as related to autokinetic movement. Journal of Experimental Psychology, 1953, 46, 171-177.

Mashrour, M. Psychophysical relations in the perception of velocity. Stockholm: Almqvist \& Wiksell, 1964. 
Royce, J. R., Carran, A. B., Aftanas, M., Lehman, R. S., \& Blumenthal, A. The autokinetic phenomenon. Psychological Bulletin, 1966, 65, 243-260.

Shaffer, O., \& Wallach, H. Extent-of-motion thresholds under subject-relative and object-relative conditions. Perception \& Psychophysics, 1966, 1, 447-451.

Strelow, E. R., \& DAY, R. H. Aftereffect of visual movement: Storage in the absence of a visual surround. Perception \& Psychophysics, 1971. 9. 485-486.

Strelow, E. R., \& DAY, R. H. Visual movement aftereffect: Evidence for independent adaptation to moving target and stationary surround. Vision Research, 1975, 15, 117-121.

Wallach, H. Perception of motion. Scientific American, 1959, 201, 56-60.

\section{NOTES}

1. Both informal observations and a control experiment showed that ISRM occurs as strongly when the motor driving the frame stopped simultaneously with frame occlusion. In the experiment, four subjects observed with the motor running throughout the trial and four when it was stopped at the moment the frame was occluded. There was no difference in ISRM between the two groups. The difference in induced-movement velocity between the two paths was significant, as in Experiment III.

2. Unpublished results on the effect of frame parameters show that, over a wide range, frame size does not affect IM.

(Recived for publication July 23, 1975; revision received February 27, 1976.) 